\title{
Infarto cerebral secundario a trombosis de la carótida interna por traumatismo cervical*
}

\author{
Cerebral infarction secondary to internal carotid thrombosis \\ following cervical trauma
}

\section{Resumen}

Se presenta el caso de una trombosis postraumática de la arteria carótida interna en un varón de 33 años, tras recibir un golpe con un balón en el cuello. La muerte se produjo 10 días después del golpe como consecuencia de un cuadro de hipertensión intracraneal y herniación cerebral secundaria a infarto isquémico extenso que afectaba a todo el territorio de la arteria cerebral media derecha, tanto superficial como profundo.

Palabras clave: Carótida interna. Traumatismo. Trombosis. Disección. Autopsia. Patología forense.

\section{Abstract}

In this paper, a case of post-traumatic thrombosis in the internal carotid artery after a blow with a ball in the neck of a 33 -year-old male is presented. The death came 10 days after the coup as a result of intracranial hypertension and cerebral herniation secondary to ischemic infarction affecting the entire territory of the middle right cerebral artery, both superficial and profound. Macroscopic and microscopic findings that largely explain the mechanism of vascular injury with intimal dissection in the proximity of an atheroma plaque located above the carotid bifurcation are discussed.

Key words: Internal carotid artery. Traumatism. Thrombosis. Dissection. Autopsy. Forensic pathology.

* Comunicación presentada a la Reunión del Club de Autopsias. XXV Congreso de la SEAP, XX Congreso de la SEC, I Congreso de la SEPAF. Zaragoza 18-21 de mayo de 2011.

\section{Introducción}

Las lesiones contusas de las arterias carótidas son poco comunes y con frecuencia pasan desapercibidas en un primer examen, de ahí la importancia de la sospecha y diagnóstico precoz ${ }^{1}$.

El comienzo de los síntomas neurológicos en los pacientes con oclusión o estenosis de la arteria carótida interna por un traumatismo cerrado puede ser inmediato, dentro de las primeras 24-48 horas, o más tardío (días o semanas después del traumatismo) ${ }^{2}$.

La mayoría de las lesiones traumáticas de la porción extracraneal de la carótida interna están causadas por un traumatismo directo penetrante, como un disparo por arma de fuego, aunque un pequeño grupo se debe a contusiones, generalmente en el contexto de un traumatismo craneal ${ }^{3}$. La protección de los vasos del cuello a cargo de las partes blandas y estructuras óseas puede ser vulnerada por varios mecanismos: flexo-extensión cervical forzada, traumatismos directos y fracturas mandibulares, cervicales y de la base del cráneo ${ }^{4}$.

En este trabajo presentamos el caso de una trombosis postraumática de la arteria carótida interna en un adulto joven, tras recibir un golpe con un balón en el cuello. La muerte se produjo 10 días después del golpe como consecuencia de un cuadro de hipertensión intracraneal y herniación cerebral secundaria a infarto isquémico extenso que afectaba a todo el territorio de la arteria cerebral media derecha, tanto superficial como profundo.
A. Rico ${ }^{1}$
M. Santos ${ }^{1}$
R. Marín ${ }^{1}$
M. Blanco ${ }^{1}$
A. Sánchez ${ }^{1}$
R. González-
Cámpora $^{2}$
J. Lucena ${ }^{1}$

${ }^{1}$ Servicio de Patología Forense. IML de Sevilla. (España).

${ }^{2}$ Servicio de Anatomía Patológica.

HU Virgen Macarena. Sevilla (España).

Correspondencia: Dr. Antonio Rico García. E-mail: anricoga@gmail.com

Fecha de recepción: 23.MAY.2011

Fecha de aceptación: 08.JUN.2011 
Figura 1.

Infarto isquémico en estadio en el territorio de la arteria cerebral media derecha. Se aprecia cerebro de respirador

con reblandecimiento cerebral generalizado y mala delimitación entre corteza y sustancia blanca.

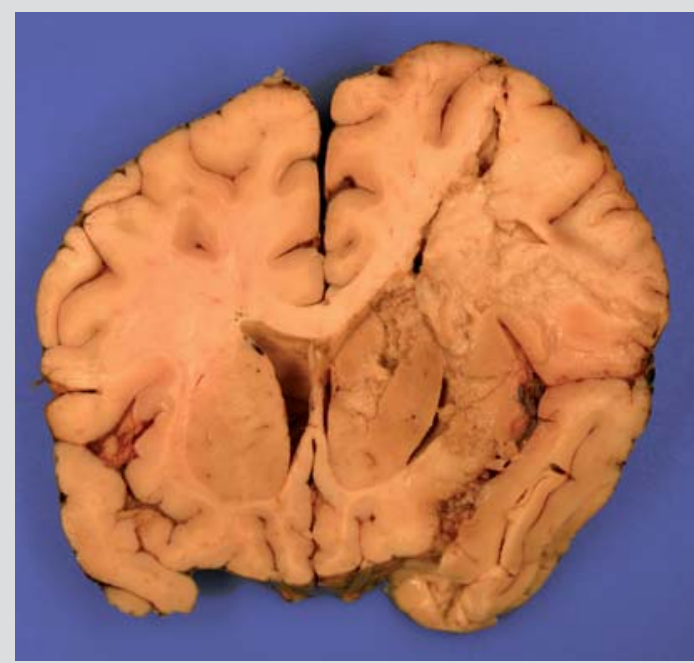

Figura 2. Infarto cerebral con rarefacción de la sustancia blanca, exudado de polimorfonucleares neutrófilos, macrófagos perivasculares y edema cerebral (H\&E).

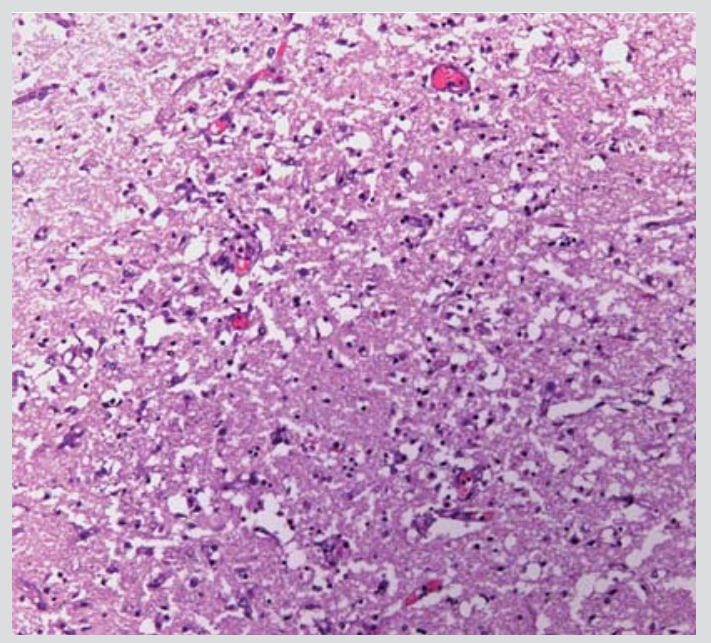

Figura 3. Corte a nivel del vermis cerebeloso Se aprecia aspecto macroscópico de muerte encefálica con necrosis de la corteza cerebelosa.

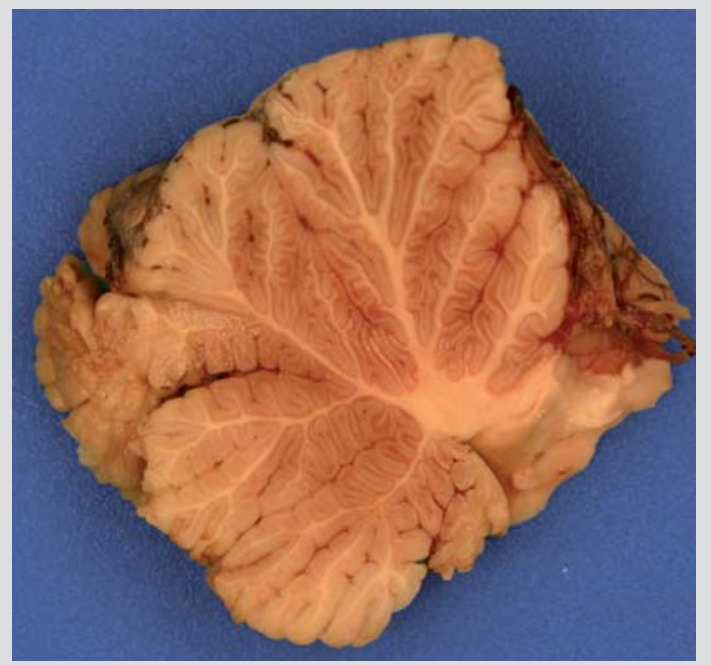

\section{Exposición del caso}

\section{Antecedentes}

Varón de 33 años, sin antecedentes patológicos conocidos, que ingresó en un servicio de urgencias hospitalario a las $7.43 \mathrm{~h}$, procedente de su domicilio, con el diagnóstico de "traumatismo craneal ocasionando ictus isquémico". La tarde anterior había sufrido un golpe con un balón en el cuello mientras jugaba un partido de fútbol como portero.

Durante su ingreso se practicó TAC craneal, que puso de manifiesto un infarto isquémico en el territorio de la arteria cerebral media derecha como consecuencia de una disección traumática de la arteria carótida interna derecha.

Presentó hipertensión endocraneal y herniación cerebral con evolución hacia muerte encefálica, por lo que entró en programa de donación de órganos falleciendo 10 días después de su ingreso.

\section{Hallazgos de autopsia}

\section{Examen externo:}

Peso: $74,9 \mathrm{~kg}$. Talla: $176 \mathrm{~cm}$ (IMC: $24,3 \mathrm{~kg} / \mathrm{m}^{2}$ ). Perímetro abdominal: $85 \mathrm{~cm}$.

En la superficie externa del cadáver se apreciaron las siguientes lesiones:

- Incisión quirúrgica en región fronto-parietotemporal derecha.

- Incisiones quirúrgicas en tórax, abdomen y ambos muslos (extracción de órganos para posterior trasplante).

\section{Examen interno:}

- Craneotomía descompresiva en región parietotemporal derecha con ausencia de calota y meninge. Encéfalo aumentado de peso (1635 g en fresco) con aspecto macroscópico de muerte cerebral. Se dejó en fijación prolongada y posteriormente se realizaron cortes coronales según técnica neuropatológica clásica. Se apreció infarto isquémico en estadio II que afectaba a todo el territorio de la arteria cerebral media derecha (tanto superficial como profundo) (Figuras 1-3).

- Trombosis que afectaba a la arteria carótida interna derecha intracraneal y se continuaba por la arteria cerebral media derecha, dentro de la cisura de Silvio (Figura 4). 
- Herniación de amígdalas cerebelosas.

- Se disecaron los troncos supraaórticos, observándose una trombosis masiva en la arteria carótida interna derecha que se iniciaba a $0,5 \mathrm{~cm}$ de la bifurcación de la arteria carótida primitiva (Figura 5).
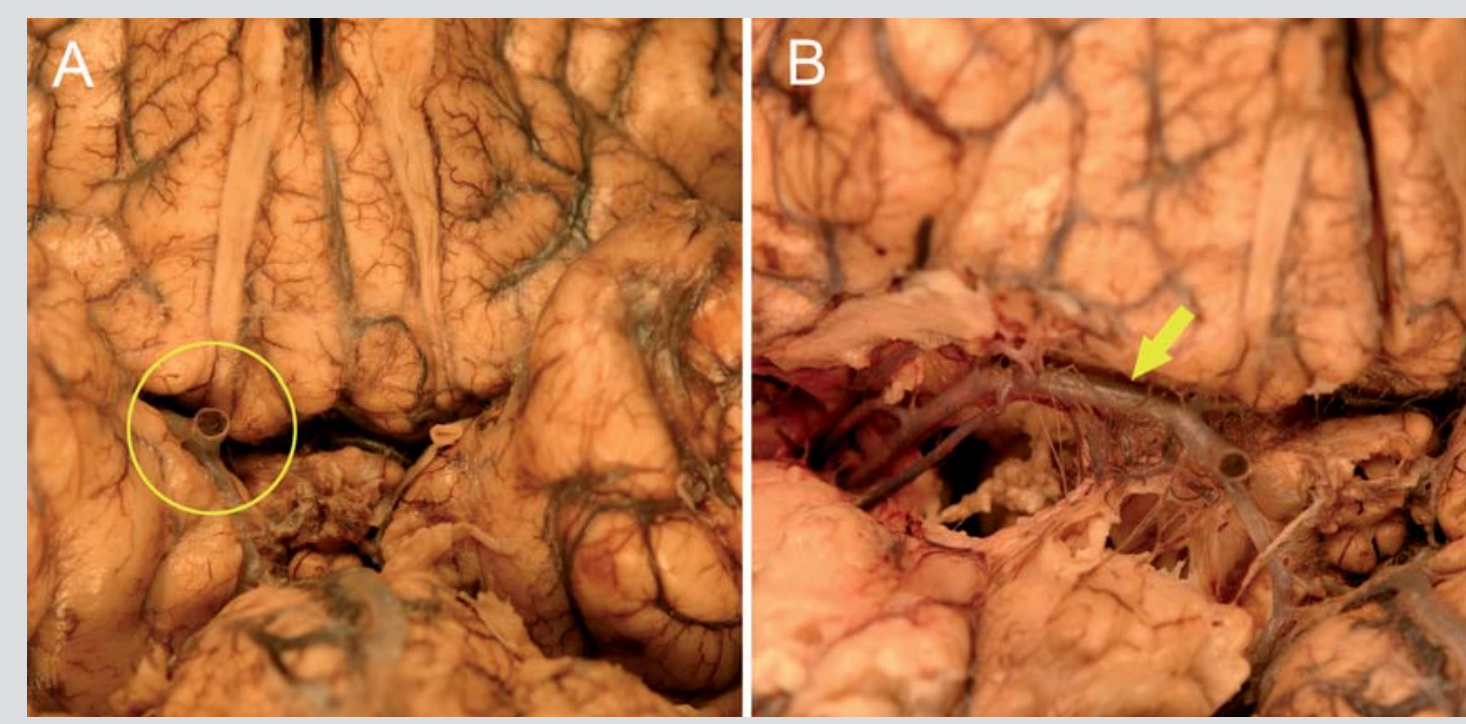

\section{Figura 4a.}

Trombosis de la arteria carótida interna derecha intracraneal (círculo).

\section{Figura $4 b$.}

La trombosis continúa por

la arteria cerebral media

derecha, dentro de la cisura de Silvio (flecha).
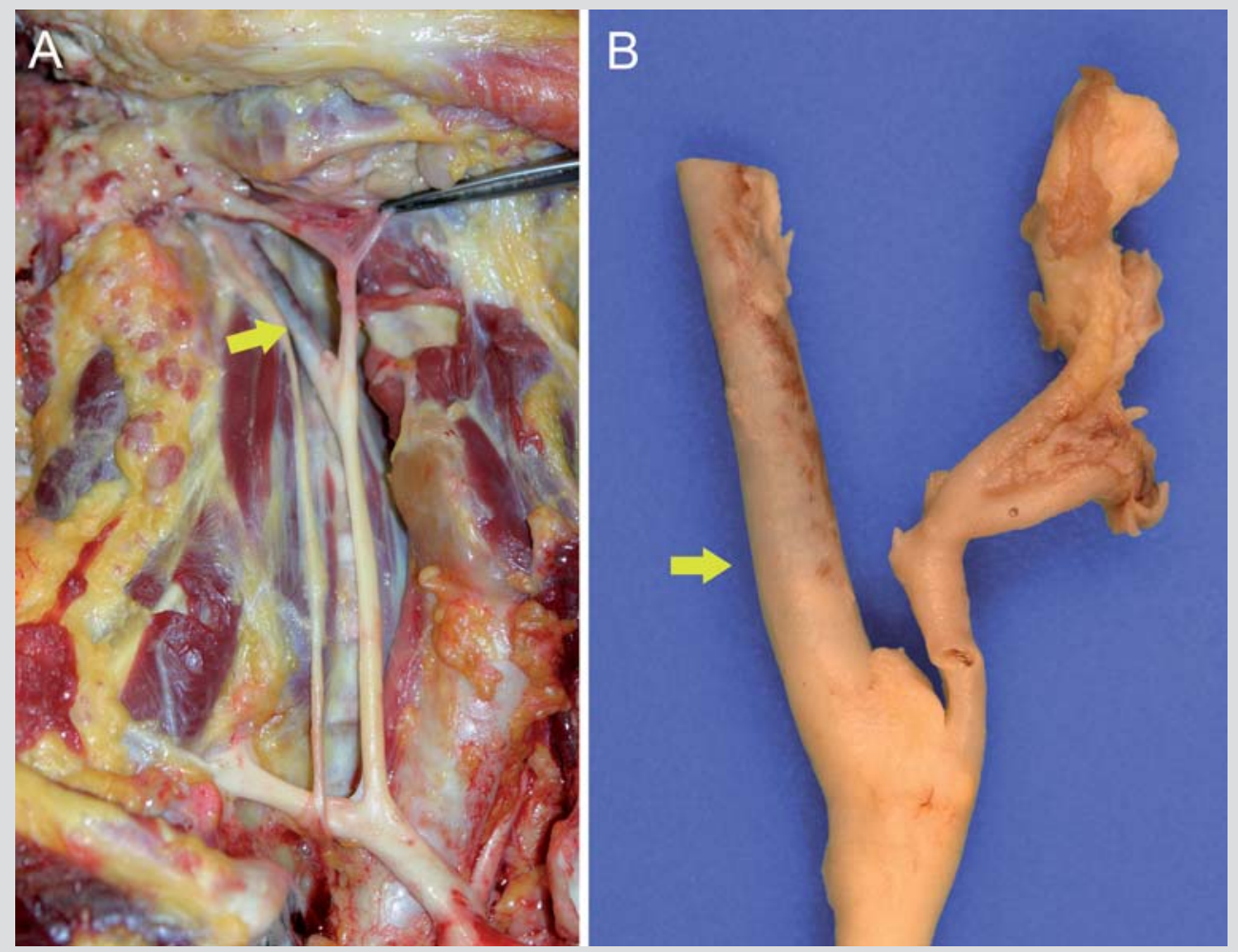

Figura $5 a$.

Disección de los troncos supraaórticos observándose una trombosis masiva en la arteria carótida interna derecha (flecha).

Figura $5 b$.

Imagen de la trombosis post-fijación (flecha). 

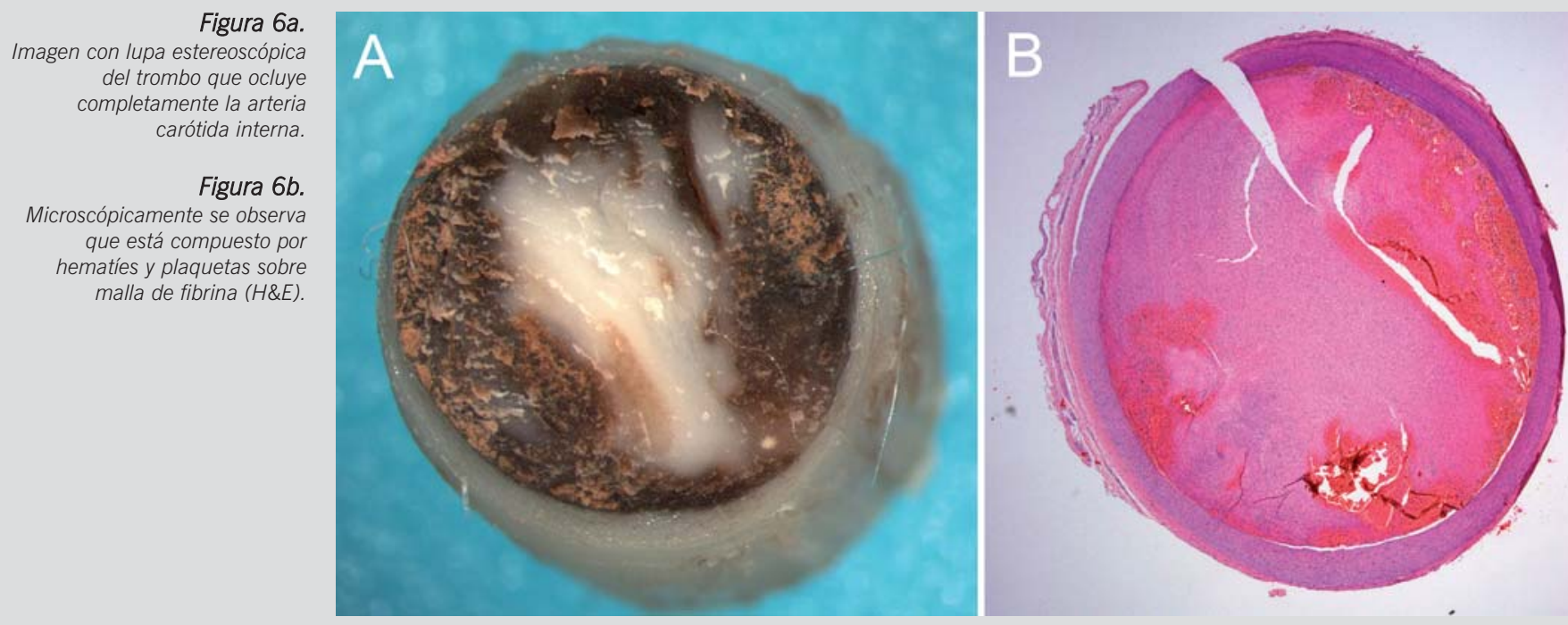

Figura $7 a$. Placa de ateroma situada por encima de la bifurcación

carotidea.

Figura $7 b$

Microscópicamente se aprecian células espumosas, macrófagos $y$ linfocitos (H\&E).
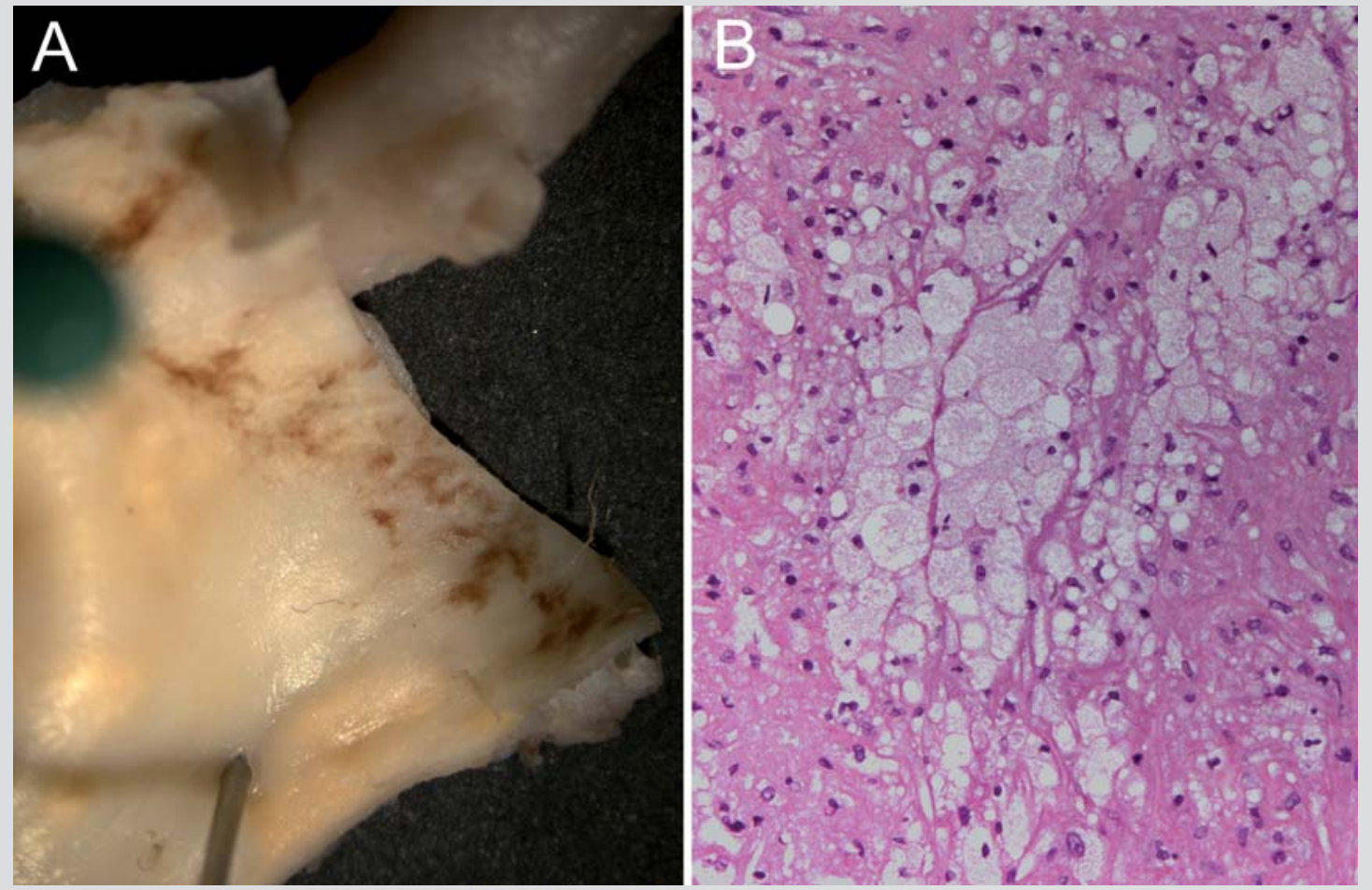

- La trombosis continuaba hasta la porción intracraneal de la arteria. Se realizaron cortes transversales y se comprobó que el trombo tenía características de vitalidad (Figura 6).

- Después de examinar la pared interna de la arteria se observó una placa de ateroma locali- zada en la bifurcación carotidea (Figura 7). Por encima de ella se apreciaba una pequeña disección de la íntima sobre la que se había iniciado la trombosis masiva (Figura 8 ). El resto de los troncos supraaórticos no presentaba anomalías. 

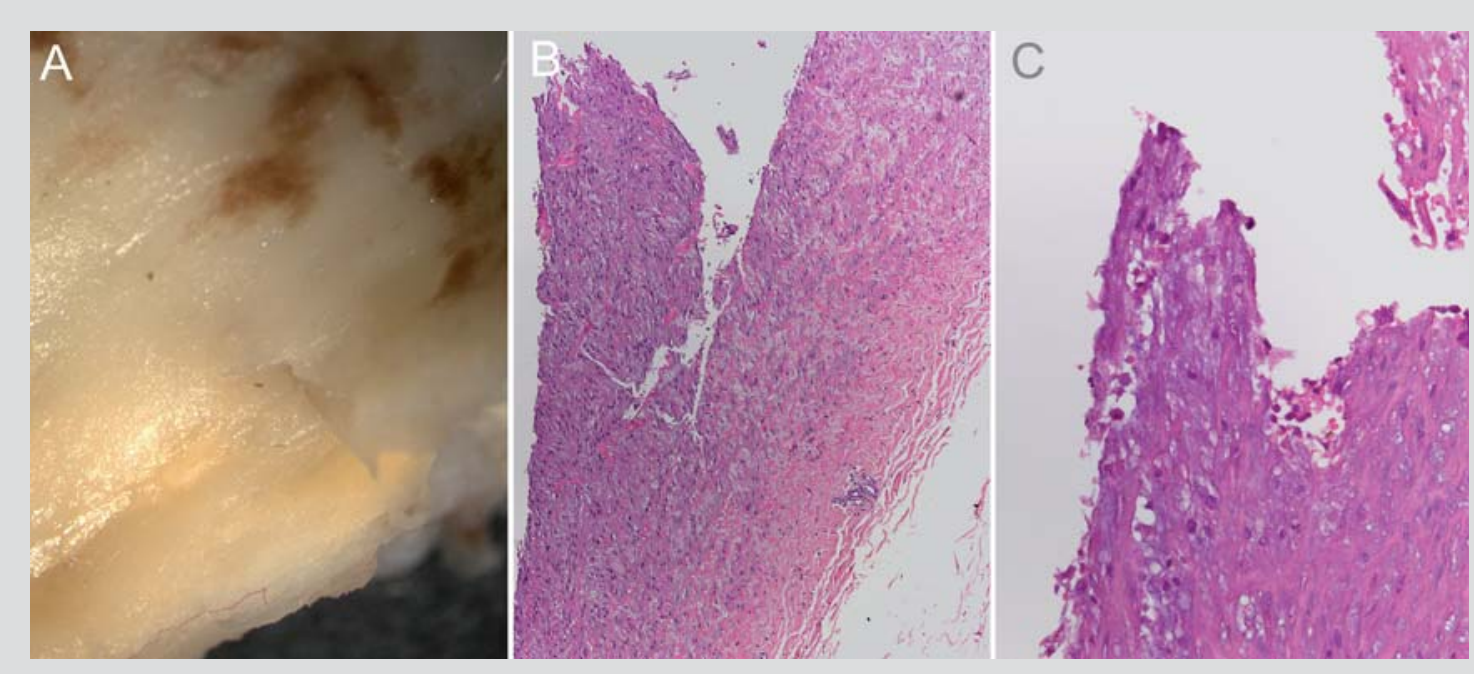

Figura $8 a$.

Disección traumática de la carótida interna con despegamiento de la íntima y formación de una "solapa" o "bolsillo" (flap).

\section{Figura $8 b$.}

Imagen microscópica de la disección de la íntima con formación del flap e infiltrado de hematíes (H\&E).

\section{Figura 8c.}

Imagen microscópica de la disección de la íntima con formación del flap e infiltrado de hematíes (H\&E).

\section{Discusión}

La incidencia de la lesión postraumática de la arteria carótida es de aproximadamente 1 por cada 1.000 pacientes que requieren ingreso hospitalario por traumatismos craneofaciales. Presenta tasas variables de mortalidad (5-40\%) y una elevada morbilidad neurológica (entre el 40 y el $80 \%)^{4}$.

Biffl, et al. (1999) ${ }^{5}$ clasificaron las lesiones arteriales carotideas postraumáticas en 5 grados, según la gravedad de las mismas:

- Grado I: lesión de la íntima o disección con estenosis $<25 \%$.

- Grado II: disección o hematoma intramural con estenosis luminal $>25 \%$, trombo intraluminal o flap intimal.

- Grado III: pseudoaneurisma.

- Grado IV: oclusión.

- Grado V: transección, observable por la extravasación de contraste radiológico.

Según el grado de la lesión, el cuadro clínico puede ser: a) asintomático; b) provocar síntomas menores; c) característico de un típico accidente cerebrovascular grave; d) la muerte del paciente ${ }^{6}$.

Los mecanismos propuestos de la lesión de la carótida interna incluyen: a) hiperextensión del cuello con rotación contralateral de la cabeza (accidentes de tráfico, prácticas deportivas, ahorcadura); b) golpe directo en el cuello; c) traumatismo intraoral; d) frac- tura de base de cráneo con lesión de la porción intrapetrosa del vaso; e) masaje carotideo diagnóstico o terapéutico; y f) maniobras de estrangulación ${ }^{2,7}$.

La hiperextensión y rotación del cuello provocan un estrechamiento y compresión de la carótida interna contra las estructuras laterales de las vértebras cervicales C1 o C2 determinando rotura en la íntima o un hematoma en la media dando lugar a trombosis y oclusión del vaso ${ }^{3}$.

Dos aéreas son más frecuentemente afectadas, el segmento cervical de la carótida interna, normalmente de 1 a $3 \mathrm{~cm}$ por encima de la bifurcación y la porción del vaso cercana a la base del cráneo. La patogénesis de la oclusión o estenosis traumática del vaso se debe la rotura de la íntima con la subsiguiente elevación de una "solapa" o "bolsillo" (flap) por la fuerza de la circulación sanguínea. Dicha rotura provoca la afluencia de plaquetas en la subíntima. La propagación del trombo tanto de forma proximal como distal puede llegar a la completa oclusión del vaso. Secundariamente a la formación del trombo local, la extensión o la embolización a la circulación cerebral puede dar lugar a graves secuelas neurológicas o la muerte. La placa arteriosclerótica predispone al desarrollo de desgarros de la íntima, pero la existencia de la placa ha sido confirmada en relativamente pocos casos de los que se han descrito ${ }^{2}$.

En el caso que exponemos se produjo un golpe directo en el cuello con un balón de fútbol que determinó, probablemente, un mecanismo de hiperextensión del cuello y rotación contralateral de la cabeza de modo 
reflejo. Los hallazgos de autopsia confirman los mecanismos patogénicos recogidos en la literatura:

1. Se produjo una pequeña disección, a unos 0,5 $\mathrm{cm}$ de la bifurcación carotidea, con despegamiento de la íntima en un lugar vulnerable a estar afectado por una placa de ateroma. La presencia de abundantes linfocitos en la placa de ateroma indica que se trataba de una placa activa y, por tanto, con más riesgo de ruptura.

2. El tiempo de latencia entre el golpe y el comienzo de los síntomas neurológicos fue de unas 12 horas.

3. La formación de un trombo y su extensión a la arteria cerebral media dio lugar a un infarto isquémico extenso que provocó la muerte del paciente diez días más tarde.

\section{Conclusión}

La trombosis postraumática de la arteria carótida interna es una patología poco común. No obstante, al estar relacionada con accidentes, agresiones o prácticas deportivas, es conveniente que el médico forense conozca sus formas de presentación y hallazgos de autopsia más frecuentes, ya que tanto su mecanismo de producción como la clínica que provoca son muy variables.

\section{Bibliografía}

1. Rodríguez J, Expósito JA, Salvador J, Forastero P. Estudio de dos casos de trombosis carotídea postraumática. Rehabilitación (Madr). 2004;38:41-4

2. Tamaki T, Saito N, Node Y, Sawada K, Terramoto A Internal Carotid Artery Stenosis Due to Atherosclerotic Plaque Damage after Whiplash Injury. J Nippon Med Sch. 2006;73:154-7.

3. Prakash S, Rai S, Bedi VS, Mohan C. Traumatic thrombosis of internal carotid artery. Indian Journal of Neurotrauma (IJNT) 2007;4:45-7.

4. Morrondo P, Trabanco S, Azkárate B, Lara G, Choperena $\mathrm{G}$. Aneurisma de carótida interna y sección de arteria mandibular tras traumatismo craneofacial. Med Intensiva. 2002;26:380-2.

5. Biffl WL, Moore EE, Ofner PJ, Brega KE, Franciose RJ, Burch JM. Blunt carotid arterial injuries: implications of a new grading scale. J Trauma. 1999;47:845-53.

6. Rajz G, Simon D, Bakon M, Goren O, Zauberman J, Zibly Z, et al. Traumatic carotid artery dissection. IMAJ 2009;11:507-8.

7. Blanco J, Morte N, Hinojal R, Payne-James JJ, Jerreat $\mathrm{P}$. Delayed presentation of carotid dissection, cerebral ischemia, and infarction following blunt trauma: two cases. J Clinical Forensic Med. 2002;9:136-40 